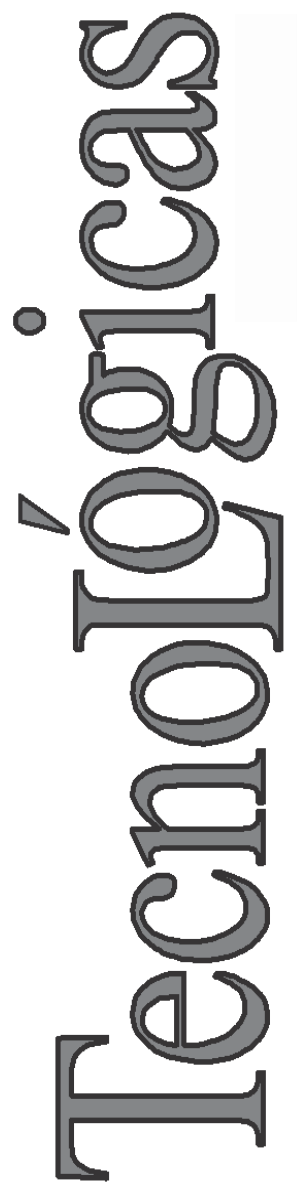

\title{
Algoritmo para un Patrón de Radiación sin Lóbulos Secundarios
}

\section{Algorithm to Achieve a Radiation Pattern without Sidelobes}

Oscar Cruz-Zamora ${ }^{1}$

Marco A. Acevedo-Mosqueda ${ }^{2}$

1 Departamento de Telecomunicaciones, Escuela

Superior de Ingeniería Mecánica y Eléctrica, México DF-México ocruzz@ipn.mx

2 Departamento de Telecomunicaciones, Escuela Superior de Ingeniería Mecánica y Eléctrica, México DF-México macevedo@ipn.mx 


\title{
Resumen
}

En este trabajo se describe el procedimiento, basado en sistema de ecuaciones de nulos direccionados para calcular los pesos necesario de un arreglo de antenas lineales, que genere un patrón de radiación (PA) con un lóbulo principal y sin lóbulos secundarios (SLS). Para lograrlo se utilizan las ecuaciones de nulos direccionados. Las ecuaciones permiten encontrar un vector de pesos, y estos pesos son ajustados en tres etapas. Las direcciones de los nulos son seleccionadas para que los lóbulos secundarios sean disminuidos y prácticamente inexistentes (hasta menos del 5 \% del valor máximo con respecto al lóbulo principal). Este procedimiento se propone como una técnicas alternativa a los formadores de haces adaptativos. Los resultados del algoritmo propuesto se comparan con el algoritmo formador de haz de mínima varianza sin distorsión (MVDR).

\section{Palabras clave}

Nulos Direccionados; algoritmo beamforming; arreglo lineal de antenas; vector de pesos; MVDR.

\begin{abstract}
This paper describes a procedure based on equations to null-steering to choose the weights required for a linear antenna array generates a radiation pattern with a main lobe and no side lobes, where the main beam is in the direction of the desired signal. This paper uses the null canceller equation. The equations are related to a weight vector, so that the weights are optimized in three adjustment process. The directions of the nulls are selected so that the side lobes are reduced and virtually nonexistent (to less than $5 \%$ of full). This procedure is proposed as an alternative to using the techniques of adaptive beamforming. In addition the results are compared with other beam-forming technique like minimum variance distortionless response (MVDR).
\end{abstract}

\section{Keywords}

Sidelobe cancellation; Beamforming; array antennas; weight vector; MVDR. 


\section{INTRODUCCIÓN}

Un arreglo de antenas es una agrupación de antenas elementales, todas ellas alimentadas en terminales comunes, que trasmiten o reciben de modo conjunto. Los elementos de un arreglo son alimentados con amplitudes y fases adecuadas de modo que los campos radiados por el conjunto proporcionan un PA deseado. El empleo de arreglos permite obtener diagramas unidireccionales estrechos, imposibles de conseguir mediante distribuciones continuas de corriente lineal, el cual, al tomar de manera natural el carácter de onda estacionaria da lugar a patrones multilobulados.

Cuando la separación entre los elementos de un arreglo lineal es equidistante, se le conoce como arreglo lineal uniforme (ULA). La Fig. 1 muestra un arreglo de $\mathrm{N}$ elementos. Donde el espacio entre los elementos del arreglo es d, y el plano de la onda llega al arreglo en una dirección de $\theta$ trasversalmente. El ángulo $\theta$ es medido en sentido de las manecillas del reloj, se le conoce como dirección de llegada de la señal recibida (AoA).

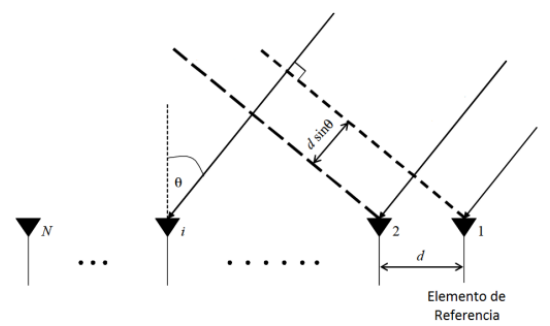

Fig. 1. Arreglo lineal simétrico uniforme. Fuente: Fakhrul 2010

La señal de llegada puede escribirse como:

$\tilde{x}_{1}(t)=A_{1}(t) \operatorname{Cos}\left\{2 \pi f_{c} t+\gamma(t)+\beta\right\}$

Dónde: $\boldsymbol{A}(\boldsymbol{t})$ es la amplitud de la señal, $\boldsymbol{f}_{\boldsymbol{c}}$ es la frecuencia de portadora, $\gamma(\mathrm{t})$ es la información, y $\boldsymbol{\beta}$ es la fase.

Si la frecuencia portadora $f_{c}$ es grande en comparación con el ancho de banda de la señal que incide, la señal puede ser tratada como cuasi-estática durante intervalos de tiempo del orden $\tau$; para la señal en la segunda antena: 
$\tilde{x}_{2}(t)=A(t) e^{j\left\{-2 \pi f_{c} \tau+\gamma(t)+\beta\right\}}=x_{1}(t) e^{j\left\{-2 \pi f_{c} \tau\right\}}$

Por lo tanto, la envolvente compleja de la señal recibida en ienésima $(\mathrm{i}=1,2, \ldots, \mathrm{N})$ elemento puede ser expresado como:

$x_{i}(t)=x_{1}(t) e^{-j\left\{2 \pi \frac{d}{\lambda}(i-1) \sin \theta\right\}}$

Definir un vector columna cuyos elementos contienen la señal recibida en su correspondiente elemento del arreglo, como:

$x(t)=\left[\begin{array}{llll}x_{1}(t) & x_{2}(t) & \ldots & x_{N}(t)\end{array}\right]^{T}$
$a(\theta)=\left[\begin{array}{llll}1 & e^{-j\left\{2 \pi \frac{d}{\lambda} \sin \theta\right\}} & \ldots & e^{-j\left\{2 \pi \frac{d}{\lambda}(N-1) \sin \theta\right\}}\end{array}\right]^{T}$

Donde $\mathbf{T}$ es la transpuesta, y $\mathbf{a}(\boldsymbol{\theta})$ es conocido, como el vector de respuesta del arreglo, el cual está en función de AoA, la respuesta de los elementos individuales, la geometría del arreglo, y la frecuencia de la señal. Supongamos que, para el rango de funcionamiento de la frecuencia portadora, el vector de respuesta no cambia. Como hemos definido la geometría en ULA. El vector de la señal recibida se puede escribir:

$\bar{x}(t)=\bar{a}(\theta) x(t)$

Por otro lado, Beamforming es la técnica de procesamiento espacial más común aplicada a un arreglo de antenas en los sistemas de comunicaciones celulares, en donde, las señales deseadas e interferencia se originan en distintas ubicaciones espaciales. Esta separación espacial es explotada por un formador de haz, que puede ser considerado como un filtro, que separa en el espacio la señal deseada, de las interferencias. Las señales de los diferentes elementos de la antena, son ponderadas, y sumadas para obtener una señal de calidad óptima. La Fig. 2 ilustra la idea de un formador de haz de banda estrecha. Si tenemos en total $\mathrm{K}$ señales, con ángulos distintos de llegada, que inciden en un arreglo de antenas 
de $\mathrm{N}$ elementos; el vector de la señal recibida puede ser escrito como (7):

$$
x(t)=\sum_{i=1}^{K} s_{i}(t) a\left(\theta_{i}\right)+n(t)
$$

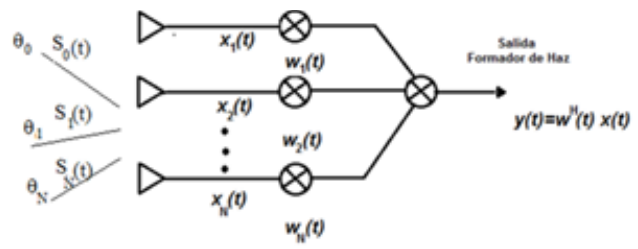

Fig. 2 Diagrama del formador. Fuente: Gross 2005

Donde $\mathrm{S}_{\mathrm{i}}(\mathrm{t})$ es la señal de i-enésima con un $\mathbf{A o A}$ de $\boldsymbol{\theta}_{\mathbf{i}}, \mathbf{a}_{\mathbf{i}}(\boldsymbol{\theta})$ es el vector de respuesta para AoA de $\boldsymbol{\theta}_{\mathbf{i}}$ y $\mathbf{N}(\mathbf{t})$ es el vector de ruido. La salida del arreglo de antenas está dada por:

$$
y(t)=w^{H}(t) x(t)
$$

Donde $w^{w=}\left[\begin{array}{llll}w_{1} & w_{2} & \ldots & w_{N}\end{array}\right]^{T}$ es el $\mathrm{N} \times 1$ vector de pesos y $\mathrm{H}$ es la traspuesta Hermitiana. El vector de pesos se elige para optimizar algún criterio del formador del haz, por ejemplo, el mínimo error cuadrático medio, la máxima relación señal a ruido e interferencia, la relación máxima señal a ruido, módulo constante, de máxima verosimilitud, etc. (Alam,2002). Sabemos, que los ángulos nulos o ceros del FA son cuando:

$\theta_{\text {nulos }}=\sin ^{-1}\left(\frac{1}{k d}\left( \pm \frac{2 n \pi}{N}-\delta\right)\right) \quad n=1,2,3, \ldots$

Donde $\delta$ es la diferencia de fase eléctrica entre dos elementos adyacentes. Para los ángulos reales, el seno de $\theta$ es menor o igual a uno, por los tanto el argumento de la ecuación debe ser menor o igual a uno (Balanis, 2002). Los ángulos de los lóbulos secundarios son máximos cuando: 
$\theta_{s}=\sin ^{-1}\left(\frac{1}{k d}\left( \pm \frac{(2 n+1) \pi}{N}-\delta\right)\right) \quad n=1,2,3, \ldots$

El ancho del haz principal se define como la apertura angular del lóbulo principal medido en determinado nivel de potencia constante, y se obtiene:

$\theta_{ \pm}=\sin ^{-1}\left(\frac{1}{k d}\left(\frac{ \pm 2.782}{N}-\delta\right)\right)$

Donde $\theta_{+}$y $\theta_{-}$son los puntos de media potencia y se encuentran normalizados para un $\mathrm{FA}=0,707$. El ancho de banda de media potencia es:

$$
H P B W=\left[\begin{array}{lll}
\theta_{+} & - & \theta_{-}
\end{array}\right]
$$

Con respecto al algoritmo de mínima varianza sin distorsión (MVDR). El término "sin distorsión" se aplica cuando se desea que la señal recibida no se distorsione después de la aplicación del vector de pesos. El objetivo del método de MVDR es reducir al mínimo la varianza del ruido a la salida del arreglo de antenas. Esto suponiendo que la señal deseada, y la no deseada tengan como media el valor cero. Utilizando de referencia el arreglo de la Fig. 2 así como las (7) y (8) (Gross, 2005) tenemos:

$$
y=\bar{w}^{H} \bar{x}=\bar{w}^{H} \bar{a}_{0} s+\bar{w}^{H} \bar{u}
$$

Con el fin de asegurar una respuesta sin distorsión, entonces:

$$
\bar{w}^{H} a_{0}=1
$$

Sustituyendo (13) en (14), la salida del arreglo es:

$$
y=s+\bar{w}^{H} \bar{u}
$$


Además, si la señal no deseada tiene como media el valor cero, el valor esperado de la salida del conjunto está dada por:

$$
E[y]=s
$$

De donde se calcula la varianza para $y$ como:

$$
\begin{aligned}
& \sigma_{M V}^{2}=E\left[\left.\bar{w}^{H} \bar{x}\right|^{2}\right]=E\left[\left|s+\bar{w}^{H} \bar{u}\right|^{2}\right]=\bar{w}^{H} \bar{R}_{u u} \bar{w} \\
& \bar{R}_{u u}=\bar{R}_{i i}+\bar{R}_{n n}
\end{aligned}
$$

Donde $\boldsymbol{R}_{\boldsymbol{i} \boldsymbol{i}}$ es la matriz de correlación para las interferencias y $\boldsymbol{R}_{\boldsymbol{n} \boldsymbol{n}}$ matriz de correlación para el ruido. Se puede minimizar esta variación mediante el método de LaGrange. Ya que todos los pesos de la matriz son interdependientes e incorporando la restricción de (14) para definir un criterio de rendimiento o función de costo, que es una combinación lineal de la varianza y la restricción, tal que:

$$
J(\bar{w})=\frac{\sigma^{2} M V}{2}+\xi\left(1-\bar{w}^{H} \bar{a}_{0}\right)=\frac{\bar{w}^{H} \bar{R}_{u u} \bar{w}}{2}+\xi\left(1-\bar{w}^{H} \bar{a}_{0}\right)
$$

Donde $\xi$ es el multiplicador de LaGrange y J(w) es la función de costo. La función de costo es una función cuadrática y se puede minimizar al establecer el gradiente igual a cero.

$\nabla_{\bar{w}} J(\bar{w})=R_{u u} \bar{w}_{M V}+\xi \bar{a}_{0}=0$

Resolviendo para el valor de los pesos, llegamos a:

$$
\bar{w}_{M V}=\bar{R}^{-1}{ }_{u u} \xi \bar{a}_{0}
$$

Con el fin de resolver el multiplicador de LaGrange se puede sustituir la (24) en (30) así (Koivo \& Heikki, 2009):

$$
\xi=\frac{1}{\bar{a}_{0}^{H} R_{u u}^{-1} \bar{a}_{0}} \quad \mathrm{y} \quad \bar{w}_{M V}=\frac{R_{u u}^{-1} \bar{a}_{0}}{\bar{a}_{0}^{H} R_{u u}^{-1} \bar{a}_{0}}
$$




\section{ALGORITMO PARA UN PATRÓN DE RADIACIÓN SIN LÓBULOS SECUNDARIOS}

El objetivo básico de un Cancelador de Lóbulos Laterales es elegir pesos, de tal manera que un valor nulo o cero se coloca en la dirección de la interferencia, y un valor máximo en la dirección de interés. Consideremos un ULA de 7 elementos isotrópicos espaciados a $d=\lambda 2$. A partir de (4) obtenemos su FA que por ser elementos isotrópicos, el FA es similar al patrón de radiación (Balanis, 1997)

$F A=e^{-j 3 \sin \theta}+e^{-j 2 \sin \theta}+e^{-j \sin \theta}+1+e^{j \sin \theta}+e^{j 2 \sin \theta}+e^{j 3 \sin t}$

En la Fig. 3 se muestra el patrón correspondiente a (23), donde los peso son todos de magnitud 1, los llamaremos pesos Wi.

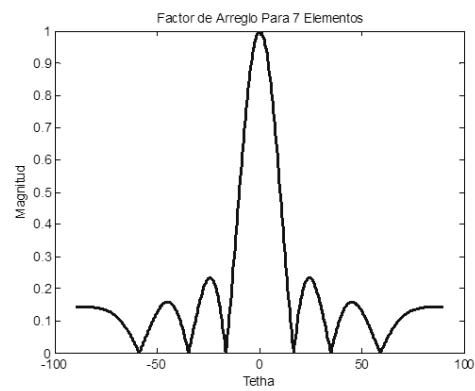

Fig. 3. Patrones de radiación de 7 elementos en distribución lineal uniforme

Utilizando (19), localizamos los máximos secundarios y proponemos semi-nulos en esas direcciones, como los mostrados en la Tabla 1:

Tabla 1. Ángulos propuestos. Fuente: Autores

\begin{tabular}{lc}
\hline Señal & Ángulo \\
\hline Deseada & $0^{\circ}$ \\
Nulo & $\pm 27,5^{\circ}$ \\
Nulo & $\pm 48^{\circ}$ \\
Nulo & $\pm 65^{\circ}$ \\
\hline
\end{tabular}


De (8) se tiene el siguiente sistema de ecuaciones, donde $\boldsymbol{w}_{\boldsymbol{i}}$ son los pesos buscados y los ángulos $\theta$ son los propuestos en la Tabla 1 .

$\left[\begin{array}{lllllll}e^{-j 3 \pi \sin \theta_{1}} & e^{-j 2 \pi \sin \theta_{1}} & e^{-j \pi \sin \theta_{1}} & 1 & e^{j \pi \sin \theta_{1}} & e^{j 2 \pi \sin \theta_{1}} & e^{j 3 \pi \sin \theta_{1}} \\ e^{-j 3 \pi \sin \theta_{2}} & e^{-j 2 \pi \sin \theta_{2}} & e^{-j \pi \sin \theta_{2}} & 1 & e^{j \pi \sin \theta_{2}} & e^{j 2 \pi \sin \theta_{2}} & e^{j 3 \pi \sin \theta_{2}} \\ e^{-j 3 \pi \sin \theta_{3}} & e^{-j 2 \pi \sin \theta_{3}} & e^{-j \pi \sin \theta_{3}} & 1 & e^{j \pi \sin \theta_{3}} & e^{j 2 \pi \sin \theta_{3}} & e^{j 3 \pi \sin \theta_{3}} \\ e^{-j 3 \pi \sin \theta_{4}} & e^{-j 2 \pi \sin \theta_{4}} & e^{-j \pi \sin \theta_{4}} & 1 & e^{j \pi \sin \theta_{4}} & e^{j 2 \pi \sin \theta_{4}} & e^{j 3 \pi \sin \theta_{4}} \\ e^{-j 3 \pi \sin \theta_{5}} & e^{-j 2 \pi \sin \theta_{5}} & e^{-j \pi \sin \theta_{5}} & 1 & e^{j \pi \sin \theta_{5}} & e^{j 2 \pi \sin \theta_{5}} & e^{j 3 \pi \sin \theta_{5}} \\ e^{-j 3 \pi \sin \theta_{6}} & e^{-j 2 \pi \sin \theta_{6}} & e^{-j \pi \sin \theta_{6}} & 1 & e^{j \pi \sin \theta_{6}} & e^{j 2 \pi \sin \theta_{6}} & e^{j 3 \pi \sin \theta_{6}} \\ e^{-j 3 \pi \sin \theta_{7}} & e^{-j 2 \pi \sin \theta_{7}} & e^{-j \pi \sin \theta_{7}} & 1 & e^{j \pi \sin \theta_{7}} & e^{j 2 \pi \sin \theta_{7}} & e^{j 3 \pi \sin \theta_{7}}\end{array}\right]\left[\begin{array}{c}w_{1} \\ w_{2} \\ w_{3} \\ w_{4} \\ w_{5} \\ w_{6} \\ w_{7}\end{array}\right]=\left[\begin{array}{c}1 \\ 0.1 \\ 0.1 \\ 0.1 \\ 0.1 \\ 0.1 \\ 0.1\end{array}\right]$

Observemos que el sistema está propuesto para que sea practicante cero (magnitud de 0,1). El (24) es resuelto por el método de Godara (2004), y se obtienen los pesos $\boldsymbol{w}_{i}$; después calculamos $\mathbf{w}_{\mathbf{i}}^{\prime}=$ $\left(\mathbf{W}_{\mathrm{i}}\right) .\left(\boldsymbol{w}_{\boldsymbol{i}}\right)$, que a esto le llamaremos herencia (ver Tabla 2).

Tabla 2. Vector de pesos primera aproximación. Fuente: Autores

\begin{tabular}{lccccc}
\hline $\begin{array}{c}\text { Pesos } \\
\text { iniciales }\end{array}$ & Magnitud & $\begin{array}{c}\text { Pesos } \\
\text { calculados }\end{array}$ & Magnitud & $\begin{array}{c}\text { Pesos } \\
\text { w'=(w)(W) }\end{array}$ & Magnitud \\
\hline W1 & 1 & $w 1$ & 0,1386 & w1' & 0,1386 \\
W2 & 1 & $w 2$ & 0,1181 & w2' & 0,1181 \\
W3 & 1 & $w 3$ & 0,1875 & w3' & 0,1875 \\
W4 & 1 & $w 4$ & 0,2116 & w4' & 0,2116 \\
W5 & 1 & $w 5$ & 0,1875 & w5' & 0,1875 \\
W6 & 1 & $w 6$ & 0,1181 & w6' & 0,1181 \\
W7 & 1 & $w 7$ & 0,1386 & w7' & 0,1386 \\
\hline
\end{tabular}

La Fig. 4 ilustra el PA de la primera aproximación con los pesos wi' (Tabla 2). Utilizando este patrón encontramos los máximos secundarios (Tabla 3), y como segundo paso, proponemos un nuevo sistema (25) en base a (8), colocando nulos o semi-nulos (magnitudes 0,05$)$.

Tabla 3. Nuevos ángulos propuestos. Fuente: Autores

\begin{tabular}{lc}
\hline Señal & Ángulo \\
\hline Deseada & $0^{\circ}$ \\
Nulo & $\pm 24^{\circ}$ \\
Nulo & $\pm 49^{\circ}$ \\
Nulo & $\pm 65^{\circ}$ \\
\hline
\end{tabular}




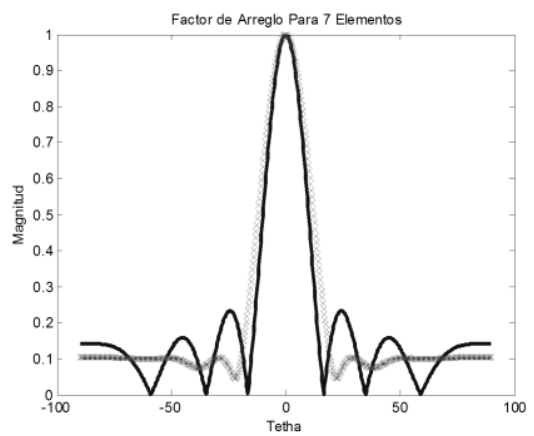

Fig. 4. Patrón de radiación para la primera aproximación. Fuente: Autores

$$
\left[\begin{array}{lllllll}
e^{-j 3 \pi \sin \theta_{1}} & e^{-j 2 \pi \sin \theta_{1}} & e^{-j \pi \sin \theta_{1}} & 1 & e^{j \pi \sin \theta_{1}} & e^{j 2 \pi \sin \theta_{1}} & e^{j 3 \pi \sin \theta_{1}} \\
e^{-j 3 \pi \sin \theta_{2}} & e^{-j 2 \pi \sin \theta_{2}} & e^{-j \pi \sin \theta_{2}} & 1 & e^{j \pi \sin \theta_{2}} & e^{j 2 \pi \sin \theta_{2}} & e^{j 3 \pi \sin \theta_{2}} \\
e^{-j 3 \pi \sin \theta_{3}} & e^{-j 2 \pi \sin \theta_{3}} & e^{-j \pi \sin \theta_{3}} & 1 & e^{j \pi \sin \theta_{3}} & e^{j 2 \pi \sin \theta_{3}} & e^{j 3 \pi \sin \theta_{3}} \\
e^{-j 3 \pi \sin \theta_{4}} & e^{-j 2 \pi \sin \theta_{4}} & e^{-j \pi \sin \theta_{4}} & 1 & e^{j \pi \sin \theta_{4}} & e^{j 2 \pi \sin \theta_{4}} & e^{j 3 \pi \sin \theta_{4}} \\
e^{-j 3 \pi \sin \theta_{5}} & e^{-j 2 \pi \sin \theta_{5}} & e^{-j \pi \sin \theta_{5}} & 1 & e^{j \pi \sin \theta_{5}} & e^{j 2 \pi \sin \theta_{5}} & e^{j 3 \pi \sin \theta_{5}} \\
e^{-j 3 \pi \sin \theta_{6}} & e^{-j 2 \pi \sin \theta_{6}} & e^{-j \pi \sin \theta_{6}} & 1 & e^{j \pi \sin \theta_{6}} & e^{j 2 \pi \sin \theta_{6}} & e^{j 3 \pi \sin \theta_{6}} \\
e^{-j 3 \pi \sin \theta_{7}} & e^{-j 2 \pi \sin \theta_{7}} & e^{-j \pi \sin \theta_{7}} & 1 & e^{j \pi \sin \theta_{7}} & e^{j 2 \pi \sin \theta_{7}} & e^{j 3 \pi \sin \theta_{7}}
\end{array}\right]\left[\begin{array}{c}
1 \\
w_{2} \\
w_{3} \\
w_{4} \\
w_{5} \\
w_{6} \\
w_{7}
\end{array}\right]=\left[\begin{array}{c}
0.05 \\
0.05 \\
0.05 \\
0.05 \\
0.05 \\
0.05
\end{array}\right]
$$

Los pesos encontrados resolviendo (25) les aplicamos la herencia para tener wi'"=(wi) $(\mathbf{w i})$, Tabla 4 :

Tabla 4. Vector de pesos segunda aproximación. Fuente: Autores

\begin{tabular}{cc}
\hline Pesos & Magnitud \\
\hline w1" & 0,0497 \\
w2" & 0,1377 \\
w3” & 0,1894 \\
w4” & 0,1965 \\
w5” & 0,1894 \\
w6” & 0,1377 \\
w7” & 0,0497 \\
\hline
\end{tabular}

El PA formado con el vector de pesos de la Tabla 4 es la Fig. 5. Para la tercera aproximación se localizan los lóbulos laterales máximos del PA de la Fig. 5, en los cuales se proponen nulos para los ángulos de la Tabla 5. Finalmente el sistema (26) es igual a cero. 


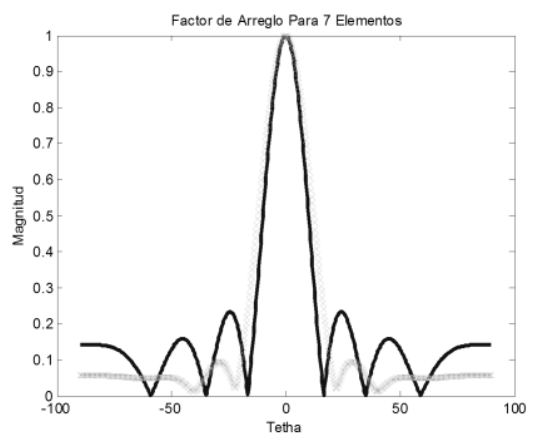

Fig. 5. Patrón de radiación del arreglo segunda aproximación. Fuente: Autores

Tabla 5. Ángulos propuestos para la tercera aproximación. Fuente: Autores

\begin{tabular}{lc}
\hline Señal & Ángulo \\
\hline Deseada & $0^{\circ}$ \\
Nulo & $\pm 29^{\circ}$ \\
Nulo & $\pm 40^{\circ}$ \\
Nulo & $\pm 65^{\circ}$ \\
\hline
\end{tabular}

$\left[\begin{array}{lllllll}e^{-j 3 \pi \sin \theta_{1}} & e^{-j 2 \pi \sin \theta_{1}} & e^{-j \pi \sin \theta_{1}} & 1 & e^{j \pi \sin \theta_{1}} & e^{j 2 \pi \sin \theta_{1}} & e^{j 3 \pi \sin \theta_{1}} \\ e^{-j 3 \pi \sin \theta_{2}} & e^{-j 2 \pi \sin \theta_{2}} & e^{-j \pi \sin \theta_{2}} & 1 & e^{j \pi \sin \theta_{2}} & e^{j 2 \pi \sin \theta_{2}} & e^{j 3 \pi \sin \theta_{2}} \\ e^{-j 3 \pi \sin \theta_{3}} & e^{-j 2 \pi \sin \theta_{3}} & e^{-j \pi \sin \theta_{3}} & 1 & e^{j \pi \sin \theta_{3}} & e^{j 2 \pi \sin \theta_{3}} & e^{j 3 \pi \sin \theta_{3}} \\ e^{-j 3 \pi \sin \theta_{4}} & e^{-j 2 \pi \sin \theta_{4}} & e^{-j \pi \sin \theta_{4}} & 1 & e^{j \pi \sin \theta_{4}} & e^{j 2 \pi \sin \theta_{4}} & e^{j 3 \pi \sin \theta_{4}} \\ e^{-j 3 \pi \sin \theta_{5}} & e^{-j 2 \pi \sin \theta_{5}} & e^{-j \pi \sin \theta_{5}} & 1 & e^{j \pi \sin \theta_{5}} & e^{j 2 \pi \sin \theta_{5}} & e^{j 3 \pi \sin \theta_{5}} \\ e^{-j 3 \pi \sin \theta_{6}} & e^{-j 2 \pi \sin \theta_{6}} & e^{-j \pi \sin \theta_{6}} & 1 & e^{j \pi \sin \theta_{6}} & e^{j 2 \pi \sin \theta_{6}} & e^{j 3 \pi \sin \theta_{6}} \\ e^{-j 3 \pi \sin \theta_{7}} & e^{-j 2 \pi \sin \theta_{7}} & e^{-j \pi \sin \theta_{7}} & 1 & e^{j \pi \sin \theta_{7}} & e^{j 2 \pi \sin \theta_{7}} & e^{j 3 \pi \sin \theta_{7}}\end{array}\right]\left[\begin{array}{l}w_{1} \\ w_{2} \\ w_{3} \\ w_{4} \\ w_{5} \\ w_{6} \\ w_{7}\end{array}\right]=\left[\begin{array}{l}1 \\ 0 \\ 0 \\ 0 \\ 0 \\ 0 \\ 0\end{array}\right]$

El proceso de herencia permite que el algoritmo SLS conserve información de un escenario previo, similares a lo que realiza los algoritmos adaptativo. El ajuste gradual de nulos $(0.1,0,05$, y 0$)$ permite que los ángulos propuestos sean útiles, para no distorsionar el PA (Tabla 6 y Fig. 6). El parámetro para detener el proceso es la relación señal a ruido; por lo que para la tercera aproximación, ya no hay mejora significativa (Tabla 7). 
Tabla 6. Vector de pesos de la tercera aproximación. Fuente: Autores

\begin{tabular}{cc}
\hline Peso & Magnitud \\
\hline w1"' & 0,0463 \\
w2"' & 0,1244 \\
w3”' & 0,2035 \\
w4"” & 0,2415 \\
w5”' & 0,2035 \\
w6”' & 0,1244 \\
w7”' & 0,0463 \\
\hline
\end{tabular}

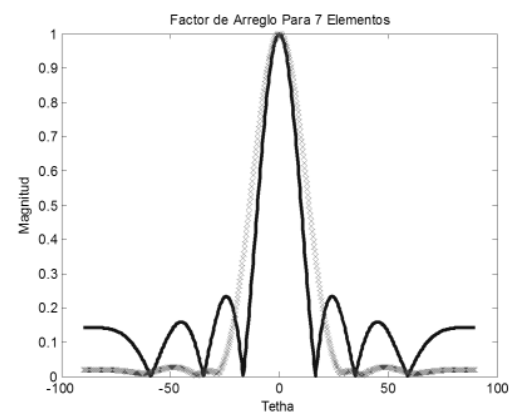

Fig. 6. Patrón de radiación sin lóbulos laterales. Fuente: Autores

Tabla 7. Relación señal a ruido para cada etapa de aproximación del método SLS. Fuente: Autores

\begin{tabular}{cccc}
\hline Etapa & 1 & 2 & 3 \\
\hline SNR dB & 2,86 & 4,92 & 22,7 \\
\hline
\end{tabular}

\section{COMPARACIÓN DEL ALGORITMO SLS vS MVDR}

Para la comparación, se consideró un arreglo de 7 elementos isotrópicos espaciados a $\mathrm{d}=\lambda 2$ en ULA; con una distribución de 6 interferencias con AoA similar a los de la Tabla 5. La Tabla 8 nos muestra una comparación de los pesos calculados por MVDR, y el algoritmo SLS, y la Fig. 7 el PA. 
Tabla 8. Vector de pesos encontrados con MVDR vs SLS. Fuente: Autores

\begin{tabular}{ccc}
\hline Pesos & MVDR & SLS \\
\hline W1 & $0,0748+0,0001 \mathrm{i}$ & 0,0463 \\
W2 & $0,1351-0,0003 \mathrm{i}$ & 0,1244 \\
W3 & $0,1788+0,0055 \mathrm{i}$ & 0,2035 \\
W4 & $0,2160+0,0015 \mathrm{i}$ & 0,2415 \\
W5 & $0,1823-0,0014 \mathrm{i}$ & 0,2035 \\
W6 & $0,1363-0,0040 \mathrm{i}$ & 0,1244 \\
W7 & $0,0767-0,0014 \mathrm{i}$ & 0,0463 \\
\hline
\end{tabular}

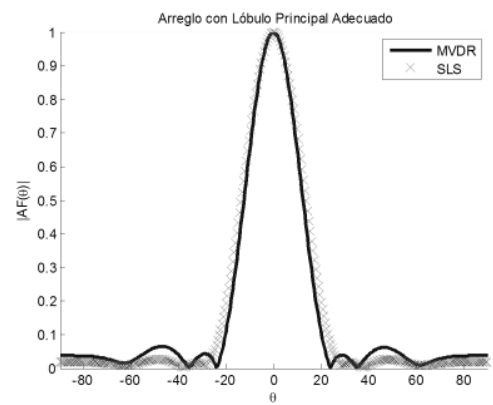

Fig. 7. Patrón de radiación SLS vs MVDR. Fuente: Autores

Tabla 9. Comparación de la SNR, HPBW para SLS, y MVDR. Fuente: Autores

\begin{tabular}{ccc}
\hline & HPBW & SNR dB \\
\hline Original & $14,80 \mathrm{o}$ & 3,12 \\
SLS & 19,960 & 22,7 \\
MVDR & 18,350 & 10,39 \\
\hline
\end{tabular}

\section{CONCLUSIONES}

Una diferencia entre SLS, y los algoritmos ya conocidos, es que estos, intentan que no exista haz en las direcciones de interferencia; el método SLS pretendes que solo exista haz en la dirección de la señal de interés. En la comparación del algoritmo SLS contra el método MVDR no se toma en cuenta la velocidad de convergencia de los algoritmos, solo se compara la SNR, para un escenario estático, es decir un grupo de interferencias con AoA, igual a los ilustrados en la Tabla 5. La Tabla 8 muestra el vector de pesos encontrado por el algoritmo MVDR vs SLS donde se puede observar que 
los pesos de SLS no son complejos. La Fig. 7 muestra el PA para el vector de pesos encontrado por MVDR, donde se observan lóbulos secundarios en 47,5o y menos 47,5o, mayores al 6\% con respecto al lóbulo principal. Sin embargo los lóbulos principales son muy similares, ya que ambos procedimientos tienden a ensanchar el haz. Para SLS el HPBW de 19,96o, y para MVDR es de 18,35o, pero la SRN es mejor para SLS con $22,7 \mathrm{~dB}$ en comparación con los 10,39 dB del método MVDR, esto debido a los lóbulos secundarios. Lo anterior nos permite concluir que el algoritmo SLS presenta mejoras con respecto al método MVDR, para un escenario previamente establecido.

\section{REFERENCIAS}

Alam Fakhrul (2002). Space Time Processing for Third Generation CDMA Systems, Thesis doctoral Universidad faculty of the Virginia Polytechnic Institute en Estados Unidos.

Balanis C.A, Bellofiore S., Foutz, R. Govindarajula, I. Bahceci. (2002). Smart antenna system analysis, integration and performance for mobile ad-hoc networks, Vol. 50, No. 5 (2).

Balanis C.A. (1997). Antenna theory: Analysis and Design. John Wiley and Sons, NewYork. (4).

Fakhrul Alam. (2010). Space Time Processing for Third Generation CDMA Systems, Thesis doctoral.

Godara. Lal Chand. (2004). Smart antennas Adaptive antennas. I. Title. II. Electrical engineering and applied signal processing, CRC Press LLC Pag. 93-159.

Gross F.B. (2005). Smart Antennas for Wireless Communication, McGraw-Hill, pp. 77, 267(3).

Koivo \& Heikki. (2009). Systems engineering in wireless communications / Heikki Koivo, Mohammed Elmusrati. John Wiley \& Sons, United Kingdom. 